\title{
Efficient Load Balancing Schema using Multiple Mobile Sink
}

\author{
S. Prabhavathi \\ Prof Dept of CSE \\ RYMEC \\ Bellary India
}

\author{
Subramanyam, $\mathrm{PhD}$ \\ Prof \& Head \\ Dept of CSE, \\ AITS Razampet ,India
}

\author{
A. Ananda Rao, PhD \\ Prof \& Dir Academic \& \\ Planning, JNTUCEA \\ Anantapur, India
}

\begin{abstract}
Ensuring efficient and fast data aggregation technique is one of the challenging task when it is considered for large-scale wireless sensor network (WSN). The problems of presence of single sink, distance between sink and cluster, as well as type of presence of dynamic task to be performed within different cluster is extremely difficult to address for reliable data aggregation technique in WSN. Therefore, the proposed system introduces a novel cylindrical topology of WSN that ensures an efficient task allocation strategy in large-scale WSN architecture. The performance of the data aggregation process is further increased by considering presence of multiple mobile sink that adds exponential benefits to the task allocation policy proposed. The results simulated in MATLAB shows satisfactory performance by considering packet delivery ratio, delay minimization, and completion time of data aggregation process
\end{abstract}

\section{Keywords}

Wireless Sensor Network, Task Allocation Policy, Wireless Topology

\section{INTRODUCTION}

The area of wireless networking system has already gained a pace to enable connectivity of higher dimension. Out of various types of wireless networking, the most commonly deployed is wireless sensor network (WSN) in commercial area. The applications of WSN consist of industrial monitoring, environmental monitoring, healthcare monitoring, and military surveillance. The wireless sensor network is characterized by interconnectivity of small or large number of sensor nodes which gathers data and forward it to another typical node called as sink node. WSN consists mainly of three types of nodes - cluster head, member node, and sink node. The cluster head and member nodes can also be termed as normal sensor nodes, where the term 'cluster head' and 'member' is assigned owing to the task they perform as shown in Fig.1. The prime task of the entire member node is to perform gathering all the physical data e.g. thermal, motion, pressure, moisture, smoke etc. and they forward them to the cluster head. Another distinction factor is that cluster node has comparatively higher residual energy as compared to member node as they need to forward a massive aggregated data to the sink. This phenomenon is also called as data aggregation, which is accompanied by two major issues e.g. i) energy depletion [1] and ii) overhead [2]. Energy depletion occurs as all the cluster heads consumes higher energy to forward the aggregated data for which purpose the average lifetime drops. While overhead is created on the sink node side as it is quite possible that some of the clusters carry similar data owing to TDMA scheduling. In addition, the sensor nodes are highly energy constrained with very limited availability of buffer and bandwidth and processing capability [3][4]. The problem becomes much worst when a very large scale WSN with dynamic traffic is considered. Owing to constraint capacity of the sensor nodes, it becomes very challenging task to manage the load and process the job in progress order with respect to time and message priority.

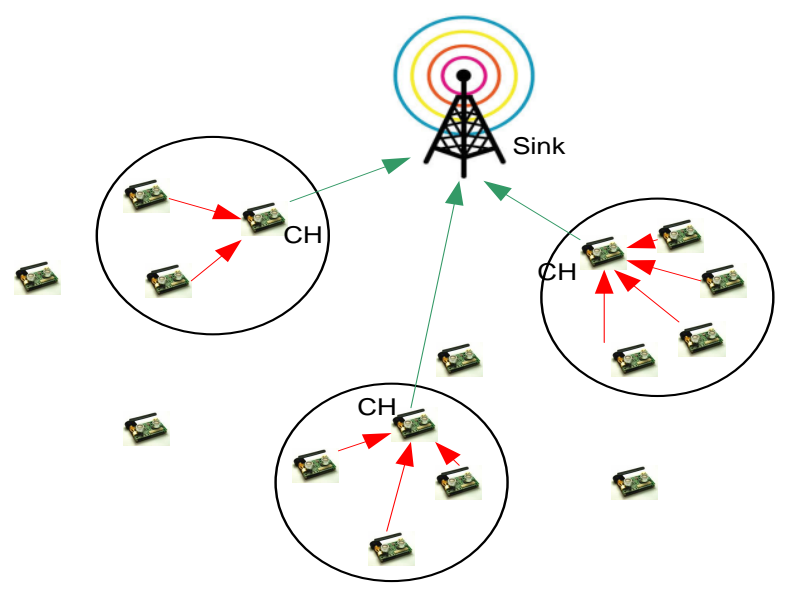

Figure 1 The Cluster Architecture

The amount of the data being aggregated by any single sensor node is quite massive and hence designed a load balancing mechanism will require a closer look into the existing topology as well as the existing communication pattern. This paper basically presents a novel model of 3 dimensional topological model termed as cylindrical topology that has attempted to mitigate the traffic abnormality, reduction in reretransmission attempt, higher reliability of processing the query task by introducing multiple mobile nodes. The proposed outcome is evaluated with respect to packet delivery ratio, delay, and task completion time to find that proposed system excels better than the existing system.

\section{2 . RELATED WORK}

Gupta [5] in their study discussed that the maximum transmission power of the nodes is used to become the cluster member. Cluster membership depends on the communication cost. The proposed approach does not consider the backup recovery. A load balanced clustering approach discussed by Zhang et al. [6] uses comprehensive weight value composed of distance between the leader and the member and the residual energy to improve cluster member choice. It also uses optimization threshold value to avoid load imbalance. The algorithm considers load equalization for creating balanced cluster. Israr and Awan [7] have proposed a multi-hop clustering algorithm for load balancing in wireless sensor networks have used layered approach for intra cluster and inter cluster communication. The algorithm consider homogeneous network. Kim have proposed a reconfiguration of cluster leader for load balancing in wireless sensor networks [8] that increases the network lifetime by fairly distributing the cluster 
leaders. Reconfiguration of the cluster is done based of the number of general nodes in the cluster $\&$ the number of cluster leaders within the cluster leader's transmission range. The algorithm provides effective data aggregation.

A novel load balancing scheduling algorithm for wireless sensor networks [8] uses optimal scheduling algorithm for packet forwarding which determines the time slot for sending the packets for the nodes. The algorithm provides uniform packet loss probability for all the nodes. The algorithm uses balanced cost objective function for optimum scheduling. Ozdemir et al. [9] have presented a secured traffic management for heterogeneous wireless sensor networks [9] that uses pseudo sink in order to improve data accuracy and bandwidth utilization of WSN to increase network lifetime. Zhang et al. [10] have presented a load balanced algorithm in wireless sensor networks based on pruning mechanism that can handle the hot point problems which uses pruning mechanism in the cluster to balance the load in the network. Evaluation function in the algorithm is based on pruning mechanism and uses nodes location, residual energy and count of cluster nodes as its parameter to find its cost.

Mahdari et al. [11] have proposed a framework for load balancing in energy efficient connected coverage wireless sensor networks that consider sensing coverage \& network connectivity by dividing the sensor network nodes into subsets. It turns on some extra nodes in each subset to ensure network connectivity. The problem with this approach is to find the existence of critical nodes. These nodes may be on all the time and the network will be partitioned if these nodes die. Canci et al [12] have addressed the energy issues in relation with Load Balancing in Sensor Networks that provides possible innetwork method for adaptive distributed control of energy consumption. Other methodologies like market based algorithm or game theoretic algorithm can be used. The algorithm assumes complete connectivity.

Low et al. [13] have presented a technique of load balancing for Wireless Sensor Networks typically targeting for cluster leaders in wireless sensor networks by considering the traffic load as the key parameter. It is assumed that the traffic load contributed by all the sensor nodes is same, which is the special case of this algorithm. In general case the algorithm is NP hard. It uses centralized approach and assumes that each node is aware of the network. Ma et al. [14] have presented a clustering and Load Balancing in Hybrid Sensor Network with mobile Cluster Nodes that consider the problem of positioning mobile cluster leaders and balancing traffic load in hybrid sensor network which consists of static and mobile nodes. It is stated that the location of the cluster leader can affect network lifetime significantly. Network load can be balanced and lifetime can be prolonged by moving cluster leader to better location.

Deng and $\mathrm{Hu}$ [15] have presented a solution for Load Balancing Clustering Algorithm exclusively for Heterogeneous Wireless Sensor Networks where the load balanced group clustering to balance the battery power in wireless sensor network by implementing dynamic route calculation according to the condition of energy distribution in the network. It makes use of heterogeneous energy to realize load balance.

Zeynali et al. [16] have presented a fuzzy Based Approach for Load Balanced Distributing database on Sensor Networks for load balanced distributing database on sensor network that prolong the network lifetime. In this algorithm vertical partitioning algorithm for distributing database on sensors is used. In this approach, first clusters are formed and then distribute partitions on clusters. In this paper, we have examined the proposed load balancing algorithms with respect to energy requirements. In wireless sensor network energy is the most valuable resource. The algorithms presented in this paper offer a promising improvement over conventional algorithms. However there is still much work to be done. Optimal clustering in terms of energy efficiency should eliminate all the overhead of cluster leader selection process as well as cluster member selection

process. Again re-clustering should be done efficiently to improve the network lifetime

\section{KEY ATTRIBUTES}

There are several key attributes that must be considered in wireless sensor networks.

- Selection of Cluster leaders and Clusters: The clustering concept offers tremendous benefits for wireless sensor networks. However when designing for a particular application, designers must carefully examine the formation of clusters in the network. Depending on the application, certain requirements for the number of nodes in a cluster or its physical size may play an important role in its operation. This prerequisite may have an impact on how cluster leaders are selected.

- Data Aggregation: One major advantage of wireless sensor networks is the ability for data aggregation to occur in the network. In a densely populated network there are often multiple nodes sensing similar information. Data aggregation allows the differentiation between sensed data and useful data. Network processing makes this process possible and now it is fundamental in many sensor network schemes, as the power required for processing tasks is substantially less than communication tasks. As such, the amount of data transferred in network should be minimized. Many clustering schemes provide data aggregation capabilities, and as such, the requirement for data aggregation should be carefully considered when selecting a clustering approach.

- Repair Mechanisms: Due to the nature of Wireless Sensor Networks, they are often prone to node mobility, node death and interference. All of these situations can result in link failure. When looking at clustering schemes, it is important to look at the mechanisms in place for link recovery and reliable data communication.

\section{4 .PROPOSED SYSTEM}

For large network, usually the sensor nodes are dropped from the airplane in adhoc fashion. For this reason, it is quite possible that all the sensor nodes are not lying in one plane from the distance of ground, which makes the topology looks like in 3 dimensional pattern inspite of 2-dimensional pattern. 


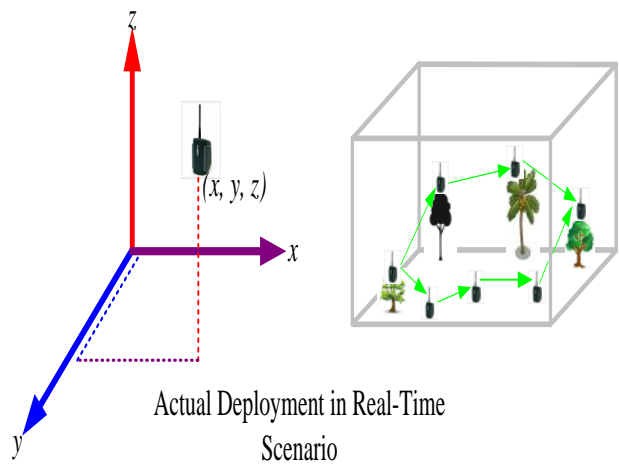

Figure 2 Considered scenario of evaluation

While dropping from the plane, some of the sensor nodes may possibly dropped at the height of some tree or some peak areas, while the possibility of existence of nodes in ground are quite high. Such real time deployment occasionally creates an issue for performing data aggregation mechanism owing to the abnormal or uncertain distances between the sensors lying on the ground and sensors lying on some height objects. We consider this scenario as it is quite challenging to perform communication, and we can expect drop of performance of data aggregation too in normal environment. Hence, we propose a very novel of performing routing considering the scenario exhibited in Fig.2.

Majority of the conventional task considers circular pattern of communication range, which is very much suited for planner deployment. However, for certain nodes with significant peak distance from the ground, the communication channel may be difficult to evaluate from traffic analysis viewpoint. This problem can be overcome, if a cylindrical based communication channel is designed in such exclusive case of node deployment as shown in Fig.3.

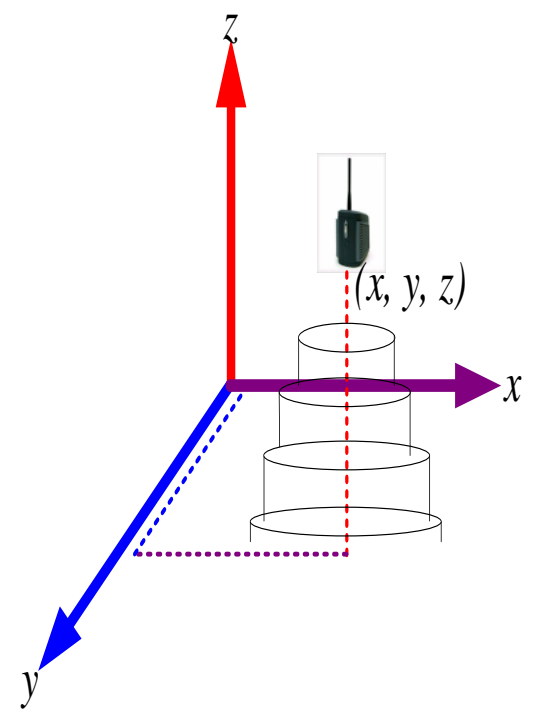

Figure3Formation of cylindrical pattern

The prime target of the proposed technique is to design cylindrical pattern based architectural framework for modeling load balancing aware system for an efficient routing in wireless sensor network that ensures very low overhead and reliable process of transmitting data packets. In order to ensure better load balancing, the system considers existence of multiple sink that area of mobile type as shown in Fig 4. The sink node determines the proper tasks to be executed by sensor nodes and then dispatches tasks to proper sensor nodes that are selected based on an allocation algorithm. The major significance of the proposed technique is that it furnishes flexibility and compatibility to large scale wireless sensor network, where allocation of task may be considered as problem scenario in dynamic scale and in such terrain region as exhibited in Fig.2. The proposed framework also intends to exhibit that presence of multiple mobile sink can saves the communication overhead that normally exist during massive data aggregation techniques when all nodes are constantly up without losing the performances compared with the previous efficient load balancing techniques especially in dense networks.

The operational requirements of the proposed system are as follows:

- A scenario of large scale WSN network deployed as discussed in Fig.2 is assumed with the presence of static nodes (Cluster Leader) and mobile nodes (Sink node). The geometrically considered cylindrical pattern in mapped in the WSN simulation area to enable the evaluation of an efficient load balancing technique.

- The proposed system should be able to deploy the nodes in random manner in the proposed topology that is again classified into i) cylindrical based region as shown in Fig.3 and ii) arc-based region for further topological computation in considered WSN.

- $\quad$ The proposed system considers a mobility model using random waypoint model for mapping the orientation of multiple mobile sinks. The proposed load balancing topology should be able to implement the routing protocols for task allocation update packages that moves along the cylindrical based region and task allocation query packages move along the arc-based region in the considered topology of WSN.

- The system should have an efficient updating process for the task allocation system and packets of information being transacted due to it.

- The system should not increase the network congestion if the proposed algorithms considering load balancing are implemented

This method assumes that nodes moves around and relay each other packet in a cylindrical pattern owing to the heights of terrain. This cylindrical structure is divided into 2 equal regions (tubular based as shown in Fig.5 and arc based as shown in Fig.6). Each region is tagged with a unique id. The proposed system assumes a nodal point in each region. Nearest nodes to this points in each region are selected as local node. The local node generates response to task allocation queries for all owner member nodes. Task allocation update packets move along the tubular-based region and task allocation query packages move along the arc-based region.

These update and query propagation method in cylindrical pattern exponentially minimizes system response time, because the query packet can traverse in only one arc-based region. One of the arc-based regions is considered as basic region in the considered topology. Task allocation policy in basic arc-based region periodically starts to send task allocation update packets in two directions (clockwise and counterclockwise as shown in Fig.8) while data aggregation 
process initiates. These packets gather updates of local nodes from each region. All packets are sent by geographical method of distribution.

A new node has to be initially authenticated by each of its neighbors to join the network. Once that has been accomplished, each packet sent by the node to its one-hop neighbor is authenticated by the neighbor using a message authentication tag. The one-hop neighbor then replaces the tag with its own authentication tag and forwards the packet to its neighbor. This next neighbor verifies the new authentication tag as coming from its immediate neighbor and the process is repeated iteratively until the packet reaches its destination. Therefore, each packet is authenticated at every hop.

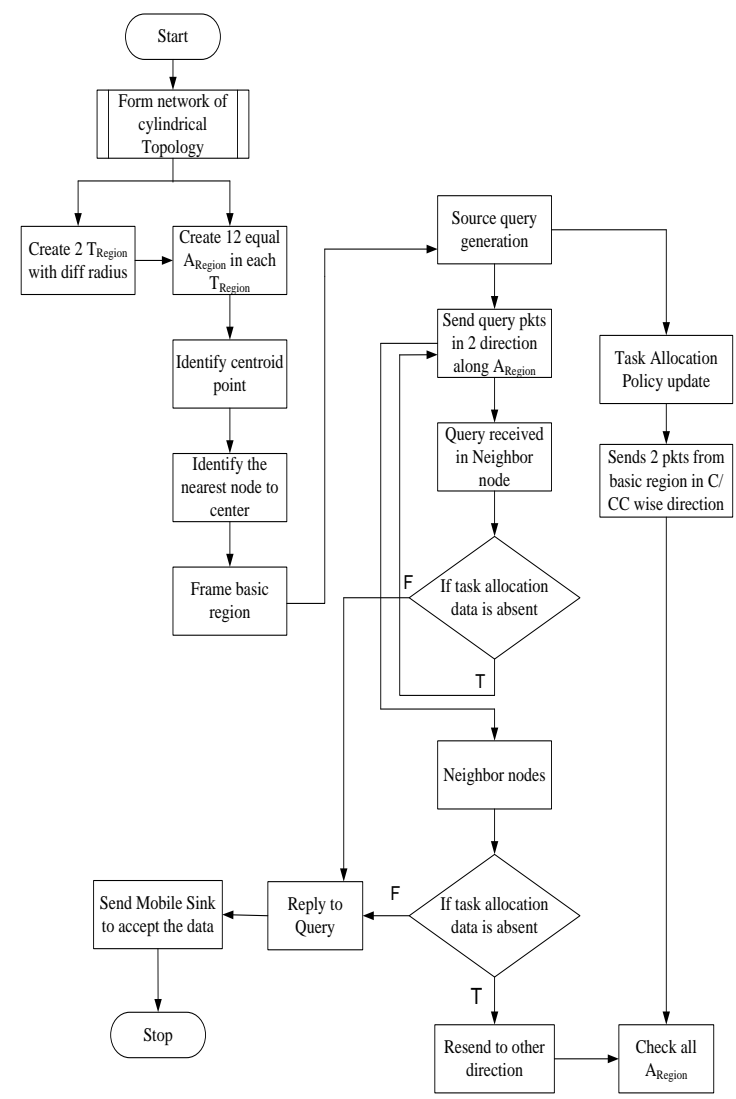

Figure 4 Proposed System Schema

\section{Module Description}

The proposed system shown in fig. 4 consists of following 3 modules:

\subsection{Cylindrical Topology Model}

Network area is modeled as a cylindrical shaped. This pattern is classified into tubular and arc based region. Each region is formed by intersection between tubular and arc based region. Each node at any moment can stay in one of this zone. Size of each region must be set so that all nodes within that region will be in radio broadcast of local node. Due to the local node are located approximately at the centroid of each zone.

In worst case, the local node may not be located in the middle of area and may be in one of the corners. Then all the nodes within the area are in task allocation policy. Each node with beaconing process can obtain direct neighbors locations. It estimates its own distance to zone centroid and other neighbor nodes too. Closest node to zone centroid point well is that load balancing node. Therefore the cluster leader of each zone will be selected

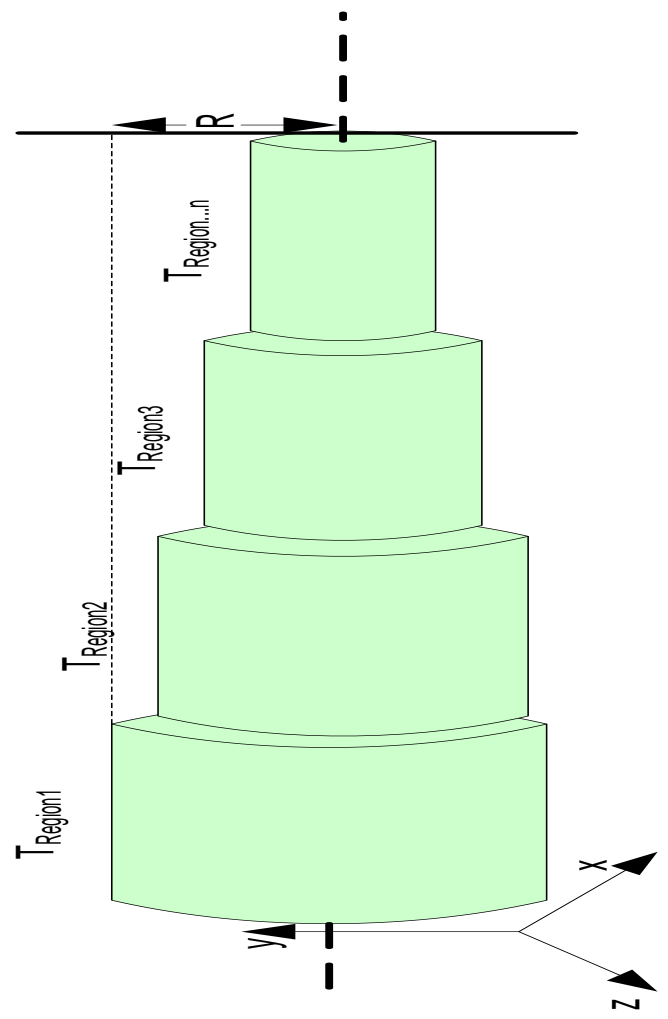

Figure 5 Tubular based Structure

\subsection{Task Allocation Update}

In the proposed work, static nodes (cluster leader) are located in centroid of the cylindrical region. One arc-based region is considered as basic starting point to start task allocation policy and its update process. Selection of basic point is predefined in network model. Task allocation update packet is sent periodically. These periods grows as the tubular-based region dimension grows Task allocation policy in each region in basic region sends two packets in clockwise and counterclockwise directions along on tubular-based region as shown in Fig.8. Each node that receive this packets if it is not sink node than it just forward packets to another mobile sink on its area. Then apply member nodes task allocation information amends and forward those to the next zone. Update packets forward continues until packets reach to the basic region again. The proposed scheme is unlike the conventional methods, so that once they attempted to gather information and then information collected will be sent. Receiving these update packets by mobile sink nodes in basic region means that all task allocation policies in tubular-based region are updated and can respond to any queries.

\subsection{Task Allocation Query}

Task allocation query process in the proposed system as shown in Fig7 is performed by sending a query packet in two different directions along the arc-based region. When a source node (cluster leader) wants to send the data to a destination node (mobile sink), if it doesn't become aware of destination's location, it should send a query packet to its neighbor nodes that may possess task allocation updates. If the neighbor node doesn't have destination position than it needs to adopt a step to find that. All the static nodes that receive the query packet, 
if they themselves have the destination's location, it replies to query and send destination's location to source node, otherwise it resend query packet to previous direction. The destination node may be located in one of the tubular-based region; therefore one of the neighbor nodes has information of destination location. So finally one of the two packets can be responded and the source node will be aware destination location as shown in Fig 9. To resist the exit of neighbor nodes from the arc-based region, the first tubular-based region (indexed as zero) and the last tubular-based region when receive query packet, don't resend it and if it cannot respond to query packet, it sends back an error message to requested node.

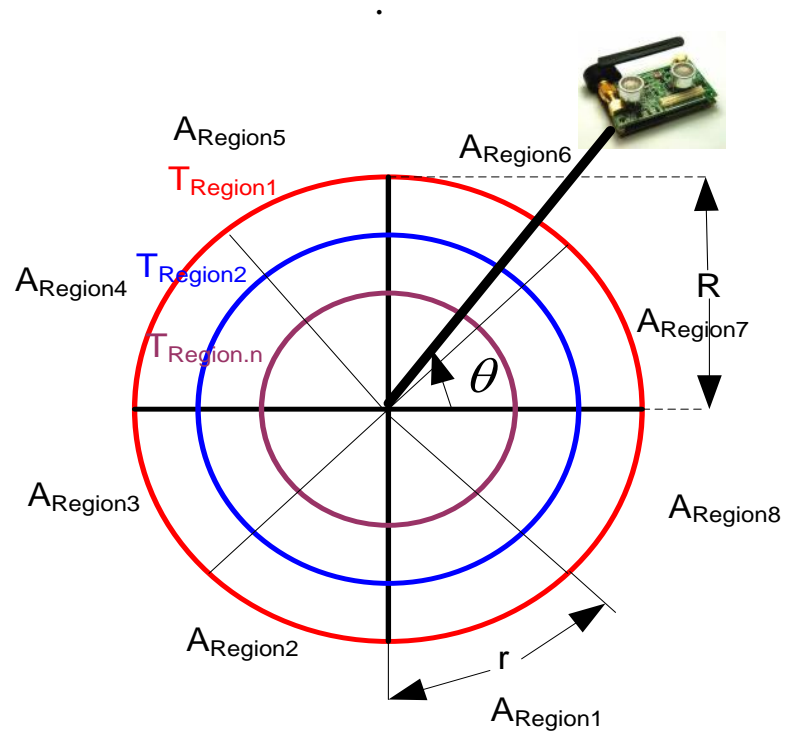

Figure 6 Task Allocation Update in proposed system

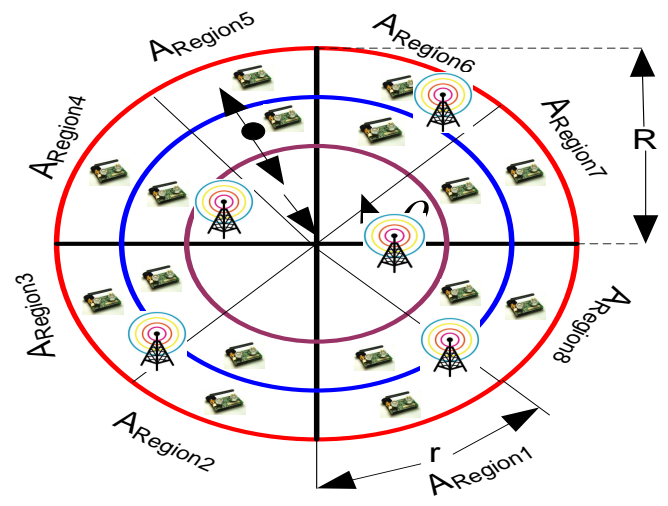

Static Sensor

(1) Mobile Sink

Figure 7 Task Allocation query process in proposed system

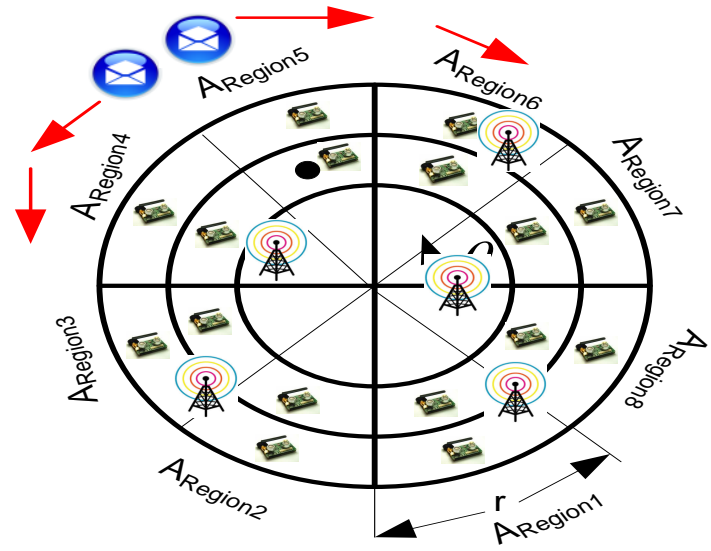

Figure 8 Message propagation in Spherical Topology

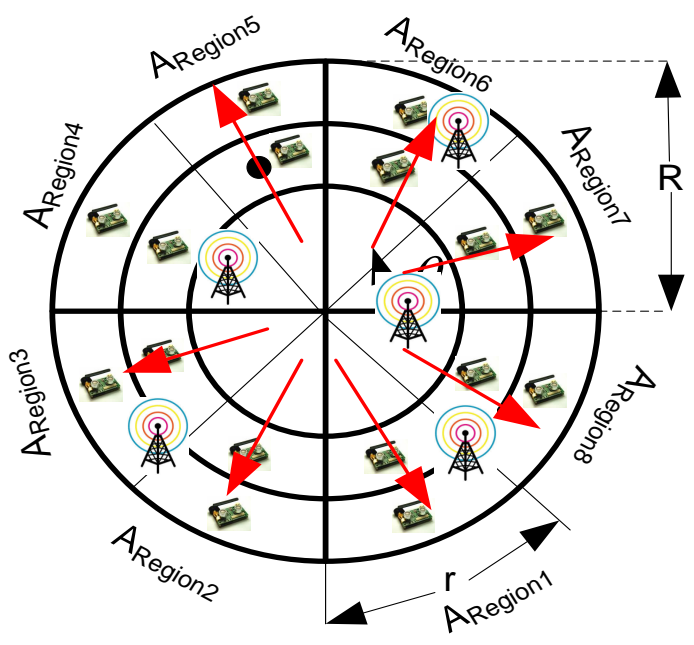

Figure 9 Final Task Allocation query process

\section{IMPLEMENTATION AND RESULTS}

The proposed system is implemented in 32 bit Windows OS with $1.84 \mathrm{GHz}$ Processor. The design environment is selected in MATLAB. The process flow chart of the implementation phase is as shown in Figure 4. The mobile nodes use IEEE 802.11 The radio and MAC model with bit rate $11 \mathrm{Mbps}$ and each radio range is approximately a disc with a 250 meter radius. The size of each simulation universe is chosen to maintain an average node density of around 100 nodes per square kilometer as shown in figure 8 . Each simulation runs for 300 simulated seconds. Each simulation runs for 300 simulated seconds. Each node moves using a random waypoint model. The node chooses a random destination and moves toward it with a constant speed chosen uniformly between one and a maximum speed $(10 \mathrm{~m} / \mathrm{s}$ unless noted otherwise). When the node reaches the destination, it chooses a new destination and begins moving toward it immediately. All simulations use a pause time of 0 second. For two location service protocols, we chose a beacon period of 2 seconds and the value update period was fixed at $25 \mathrm{~s}$.

Wireless Sensor Networks present vast challenges in terms of implementation. Clustering algorithms play a vital role in achieving the targeted design goals for a given implementation.

Algorithm: Load balancing in Spherical topology 
Input: WSN Parameters

Output: Communication completion

\section{START}

1. Consider task T1 in sub-task Mv.

2. Initiate Design of Spherical Topology in WSN

3. Compute number of tubular-based region

$\mathrm{T}_{\text {Region }}=2 \Pi \mathrm{R} / \mathrm{r}$, where $\mathrm{R}$ is network area radius and $\mathrm{r}$ is dimension of arc in each zone.

4. Compute number of arc-based region

$$
\mathrm{A}_{\text {Region }}=\mathrm{R} / \mathrm{r} \text {. }
$$

5. Compute orientation angle $\theta$ in each arc-based region.

$$
\theta=360 \mathrm{r} / 2 \Pi \mathrm{R}
$$

6. Compute expanse factor of region

$$
E_{\text {fact }}=\mathrm{N}\left(\mathrm{T}_{\text {Region }}\right), \mathrm{N}\left(\mathrm{A}_{\text {Region }}\right)
$$

Where $\mathrm{N}\left(\mathrm{T}_{\text {Region }}\right)$ signifies number of $\mathrm{T}_{\text {Region }}$ that is equivalent to divisive operator of polar coordinates $(\psi)$ by $\theta$ and $\mathrm{N}\left(\mathrm{A}_{\text {Region }}\right)$ is equivalent to divisive operator of polar length (a) by $\mathrm{r}$. It is to be noted that the system has consideration of multiple mobile sink that is assumed to find its location, and hence it can extract it polar coordinates $(\mathrm{a}, \psi)$

7. Initiate communication until all task in $\mathrm{Mv}$ is mapped.

8. For each task, find first completion time.

9. Find a task with maximum earliest completion time.

10. In TRegion, Send two packets in clock and anti-clockwise direction to find node with first completion time.

11. In ARegion, Send a query packet in 2 different direction along arc-based region.

12. Stop packet resend if it cannot respond to query packet.

\section{Complete communication}

\section{END}

For the purpose of benchmarking, the outcome accomplished from proposed system is compared with that of work done by Petrioli et al. [17] approach. Petrioli et al. [17] have discussed a framework of routing protocol that minimizes the traffic congestion using greedy algorithm on geographic based routing. The author has demonstrated some of the optimistic outcomes where it was shown that their load balancing mechanism on heavier traffic of WSN is highly resilient against localization errors The prime reason behind selecting the work of Petrioli et al. [17] for benchmarking was because the author chose to adopt a technique similar to ours. The study has considered that network topology is not required to be planer, which is similar to our assumption of dynamic terrain situation discussed in Fig.2. The metrics Packet Delivery Ratio, Delay Estimation, and Completion Time analysis is performed for task allocation strategies designed in the proposed system

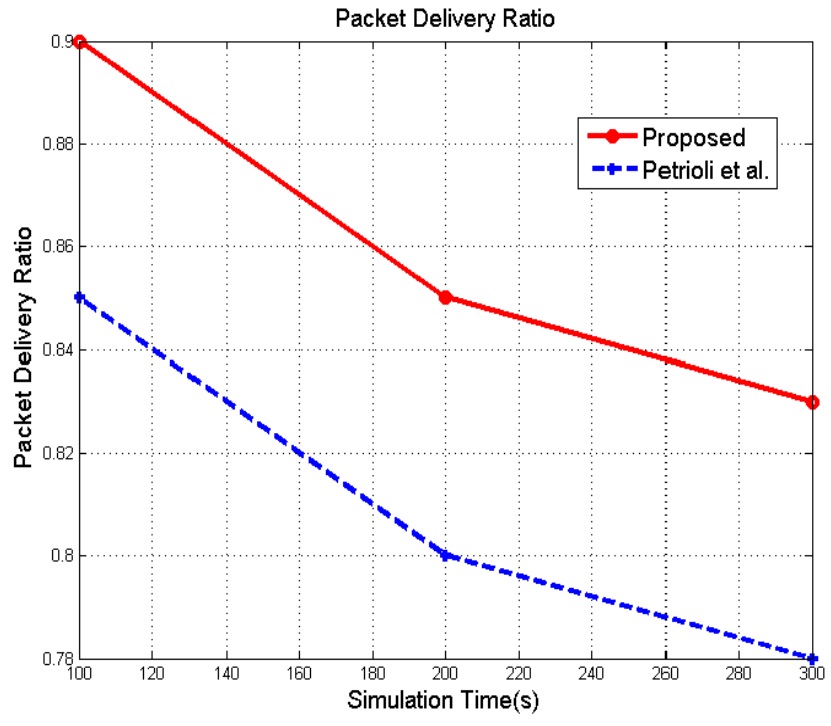

Figure 10 Packet Delivery Ratio

The packet delivery ratio will include the estimation of the number of data packets being successfully transmitted from cluster leader to the multiple mobile sink in order to perform data aggregation task. The decreasing trend of packet delivery ratio is because of the energy depletion of the sensor nodes with the progress of data aggregation cycle. The results are shown in Fig10 that exhibit that current work considering mobile sink accomplishes better packet delivery ratio as compared to the conventional technique that adopts the same system without mobile Sink node.

The packet delivery ratio recorded for the Petrioli et al. [17] work was found lesser compared to the proposed system. The prime reason behind this that Petrioli et al. [17] has no consideration of mobile sink and the load balancing mechanism highly depends on geographic based routing designed using greedy algorithm. Whereas in our case, we mitigate the congestion by increasing the higher probability of processing the task being queried using multiple sink.

Delay estimation is another parameter that has been considered to evaluate the performance of the designed system. It estimates the total time difference taken to perform the data aggregation process in common radio model (usually small scale network with all static nodes and one sink) of conventional data aggregation techniques and proposed technique. Fig11 shows that there has been an extensive delay minimization accomplishment in the proposed system that outperforms the technique considering absence of mobile sinks, which was found in study discussed by Petrioli et al. [17]. However, due to constant need of update of task allocation policy, the minimization process is somewhat slower till 200 seconds of time instant of simulation, but it rapidly accumulates the speed after 200 second. 


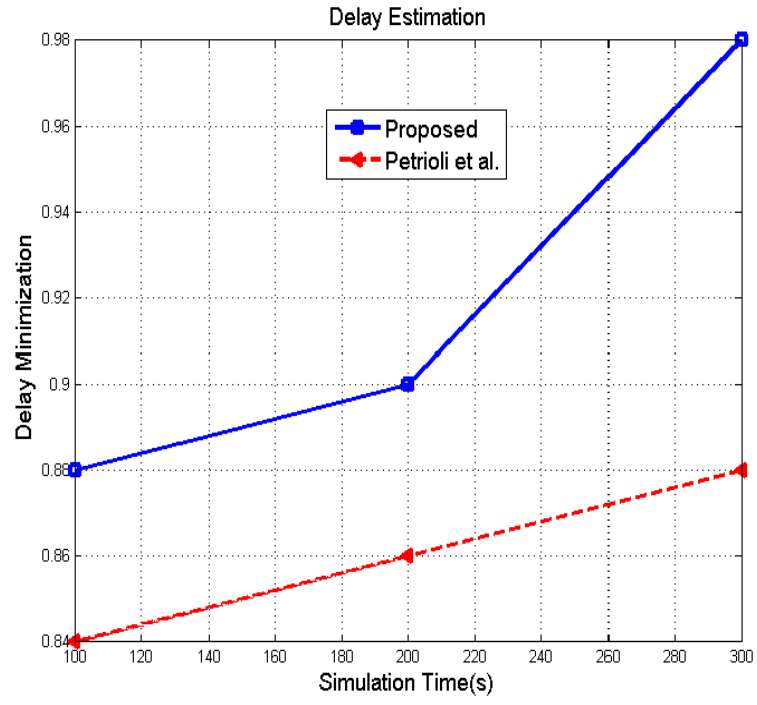

Figure 11 Evaluation of Delay Estimation

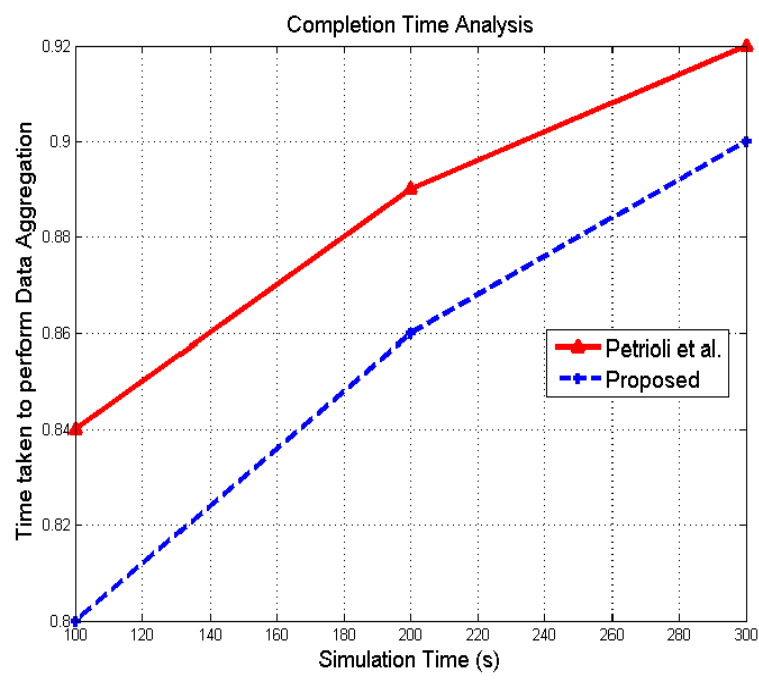

Figure 12 Completion Time Analysis

The final parameter for checking the performance evaluation is completion time. Fig12 highlights that the time taken to perform the data aggregation is quite faster in present of mobile sinks as compared conventional technique without mobile sink nodes, as discussed by the study of Petrioli et al. [17]. The query pattern is chosen to study the efficiency of the query and update mechanism of two protocols. Every node in the network initiates certain number of queries to look up the location of randomly destinations at times randomly distributed between certain intervals of time. Also, if a query is not successful, no retry is initiated. To measure the accuracy of the query reply, when the query reply is received, each source sends a single data packet of size in bytes to that destination using the replied location, this for routing done after finding a route to destination. Hence the performance is highly optimized for the proposed system.

\section{CONCLUSION}

This paper introduces an efficient technique where a scalable task allocation management is incorporated in routing for wireless sensor network that has considered cylindrical topology and multiple mobile sink. The proposed technique has got two advantages: i) Owing to presence of multiple mobile sink nodes, the data aggregation process is faster with lesser chances of circulating non-redundant packets are quite less, ii) the formulation of tubular and arc based concept addresses the discrepancies among the nodes for generating redundant query message, iii) routing reliability is ensured as the cylindrical topology furnishes the discovery of mobile sink using clockwise and anti-clockwise queries and task allocation policies. Hence, the results shows that the proposed system ensure better packet delivery ratio, delay minimization and faster completion time with less communication overhead when all nodes are constantly up without losing the performances.

\section{REFERENCES}

[1] Rauthan, J.S., Mishra, S., An Improved Approach in Clustering Algorithm for Load Balancing in Wireless Sensor Networks, International Journal of Advanced Research in Computer Engineering \& Technology Volume 1, Issue 5, July 2012

[2] Sun, K., Ning, P., Secure Distributed Cluster Formation in Wireless Sensor Networks, Proceedings of the 22nd Annual Computer Security Applications Conference, Pages 131-140, December 2006.

[3] Nazir, B., Hasbullah, H., Energy Balanced Clustering in Wireless Sensor Network, International Symposium in Information Technology (ITSim), IEEE, 2010

[4] Katiyar, V., Chand, N., Soni, S., Clustering Algorithms for Heterogeneous Wireless Sensor Network: A Survey, International Journal of Applied Engineering Research, Volume1, No 2, ISSN-0976-4259, 2010

[5] Gupta, G., Younis, M., Load-Balanced Clustering in Wireless Sensor Networks, Proceedings of International Conference on Communications, Page(s): 1848 - 1852, Vol.3, Anchorage, Alaska, May2003

[6] Zhang, H., Li, L., Yan, X., Li, X., A Load Balancing Clustering Algorithm of WSN for Data Gathering, IEEE, 2011.

[7] Israr, N., Awan, I., Multi-hop clustering Algorithm For Load Balancing in WSN, International Journal of Simulation, Vol. 8 No. 1, ISSN 1473-804x, 2006

[8] Kim, N., Heo, J., Kim, H. S., \& Kwon, W. H., Reconfiguration of Cluster head for Load Balancing in Wireless Sensor Networks, Science Direct Computer Communications, page- 153-159, 2008

[9] Ozdemir, S., Secure Load Balancing via Hierarchical Data Aggregation in Heterogeneous Sensor Networks, Journal of information science and engineering, Pages1691-1705, IEEE, 2010.

[10] Zhang, Y., Zheng, Z., Jin, Y., Wang, X., Load Balanced Algorithm In Wireless Sensor Networks Based on Pruning Mechanism, IEEE transaction, 2009.

[11] Mahdari, M., Ismail, M., Jumari, K., Load Balancing in Energy Efficient Connected Coverage Wireless Sensor Networks, IEEE Transaction, pages- 448-452, 2009

[12] Canci, C. M., Trifa, V., Martinoli, A., Threshold Based Algo. For Power Aware Load Balancing in Sensor Networks, IEEE Transaction, 2005

[13] Low, C.P., Fang, C., Mee, J. , Hang, Y.H., Load Balanced Clustering Algorithm for Wireless Sensor Networks, IEEE Communications Society, , 2007. 
[14] Ma, M., Yang, Y. , Clustering \& Load Balancing in Hybrid Sensor Network with mobile Cluster Heads, Proceedings of the 3rd international conference on quality of service in heterogeneous wired/ wireless Networks, 2006

[15] Deng, Y., Hu, Y., A Load balanced Clustering Algorithm for Heterogeneous Wireless Sensor Networks, IEEE, pages-1-4, ISBN: 978-1-4244-7159-1, 2010
[16] Zeynali, M., Khanli, L.M., Mollanejad, A., Fuzzy Based Approach for Load Balanced Distributing database on Sensor Networks, International Journal of Future Generation Communication \& Networking, Vol. 3, No. 2, June 2010 